\title{
A New Normal Form for Monodromic Nilpotent Singularities of Planar Vector Fields
}

\author{
Antonio Algaba, Cristóbal García and Jaume Ginéœ
}

\begin{abstract}
In this work, we present a new technique for solving the center problem for nilpotent singularities which consists of determining a new normal form conveniently adapted to study the center problem for this singularity. In fact, it is a pre-normal form with respect to classical Bogdanov-Takens normal formal and it allows to approach the center problem more efficiently. The new normal form is applied to several examples.
\end{abstract}

Mathematics Subject Classification. Primary 34C20; Secondary 37G05, 34C14, 37C10, 37C80.

\section{Introduction and Statement Main Results}

The center problem for planar vector fields is an important problem in the qualitative theory of the differential equation, see for instance $[5,31,33,37$, $38,42-44,47]$ and references therein.

This problem is related to the one of integrability. In fact a vector field that has a singularity of type center has a first integral of infinity class in a neighborhood of the singularity, see [39]. However, in general, it is not analytically integrable around the singularity. Therefore, it is interesting to distinguish when a vector field with a center has an analytical first integral, see $[4,5,11,25,29,32,39,40]$.

The case when a nilpotent singularity of a vector field is a center was theoretically characterized in $[5,22]$. In fact, any nilpotent system with a center is orbitally equivalent to a time-reversible system, more specifically the phase portrait is symmetric respect to the $y$-axis after a change in the time and state variables, see for instance [1,25,29].

The formal normal form for systems with a nilpotent singularity was given by Takens [46] and Bogdanov [23] showing that such systems can be formally reduced to 


$$
\dot{x}=y+a(x), \quad \dot{y}=b(x),
$$

where $a(x)$ and $b(x)$ are power series without constant and linear terms, see also [21]. To obtain the normal form (1.1) they only use changes in the variables $x$ and $y$ but no changes of time. Later using changes of time Bogdanov [24] showed that the formal orbital normal form (1.1) is reducible to

$$
\dot{x}=y+a(x), \quad \dot{y}=\sigma x^{s}, \quad \sigma= \pm 1,
$$

with $s \geq 2$. Similar results but with much simpler proofs were obtained by Sadovski [45]. The monodromy case is for $s=2 n+1$ with $\sigma=-1$ and from the normal form (1.2) it is not difficult to characterize the centers of the monodromic singularities. The monodromy of a nilpotent singularity was studied in [18], see also [16]. If the origin of system (1.2) is monodromic then it is a center if and only if $a(x)$ is an even function of $x$, see [22]. Hence, we have the following result.

Theorem 1.1. A differential system with a monodromic nilpotent singularity at the origin is orbitally equivalent to system (1.2) with $s=2 n+1, n \in \mathbb{N}$ and $\sigma=-1$, where $a(x)=\sum_{i \geq n+1} \beta_{i} x^{i},\left|\beta_{n+1}\right|<\frac{2}{\sqrt{n+1}}$. Moreover system (1.2) has a center at the origin if and only if $a(-x)=a(x)$, i.e. $\beta_{2 k+1}=0$ for all $k \in \mathbb{N}$ such that $k \geq \frac{n}{2}$. In addition, system (1.2) is analytically integrable if and only if $a(x) \equiv 0$.

In fact, this result gives a procedure to find nilpotent centers transforming the original system by a change of variables and of time to reduce the system into the normal form (1.2). However, this procedure is difficult to implement and with a high cost of computations to eliminate all the terms that not appear in the final expression of the normal form. Is for this reason that there are few families of nilpotent vector fields where the center conditions are known, see, for instance, [7,11,12,20,27]. Moreover, alternative methods are used to find such centers, as the generalized polar coordinates, Cherkas' method, approximation by nondegenerate systems, see, for instance, $[6,16,17,28-30,34,35]$. Indeed the centers of the more simple semi-quasi-homogeneous systems, that is, systems that are sum of two quasihomogeneous vector fields are not yet completely classified. The objective of this work is to find a new normal form in which we have only removed the necessary terms to solve the center problem providing a much more effective computational method. In this sense, a greater number of necessary conditions can be computed that allow to solve the problem of the center for a greater number of families of systems.

Before of showing our results, we recall the following concepts and definitions. Given $\mathbf{t}=\left(t_{1}, t_{2}\right)$ with $t_{1}$ and $t_{2}$ natural numbers without common factors, a function $f$ of two variables is quasi-homogeneous of type $\mathbf{t}$ and degree $k$ if $f\left(\varepsilon^{t_{1}} x, \varepsilon^{t_{2}} y\right)=\varepsilon^{k} f(x, y)$. The vector space of quasi-homogeneous polynomials of type $\mathbf{t}$ and degree $k$ will be denoted by $\mathcal{P}_{k}^{\mathbf{t}}$. A vector field $\mathbf{F}=(P, Q)^{T}$ is quasi-homogeneous of type $\mathbf{t}$ and degree $k$ if $P \in \mathcal{P}_{k+t_{1}}^{\mathbf{t}}$ and $Q \in \mathcal{P}_{k+t_{2}}^{\mathrm{t}}$. We will denote $\mathcal{Q}_{k}^{\mathrm{t}}$ the vector space of the quasi-homogeneous polynomial vector fields of type $\mathbf{t}$ and degree $k$. 
Any vector field can be expanded into quasi-homogeneous terms of type $\mathbf{t}$ of successive degrees. Thus, the vector field $\mathbf{F}$ can be written in the form $\mathbf{F}=$ $\mathbf{F}_{r}+\mathbf{F}_{r+1}+\cdots$ for some $r \in \mathbb{Z}$, where $\mathbf{F}_{j}=\left(P_{j+t_{1}}, Q_{j+t_{2}}\right)^{T} \in \mathcal{Q}_{j}^{\mathbf{t}}$ and $\mathbf{F}_{r} \not \equiv \mathbf{0}$. If we select the type $\mathbf{t}=(1,1)$, we are using in fact the Taylor expansion, but in general, each term in the above expansion involves monomials with different degrees.

We will denote by $\mathbf{D}_{0}=\left(t_{1} x, t_{2} y\right)^{T} \in \mathcal{Q}_{0}^{\mathbf{t}}$ (a dissipative quasi-homogeneous vector field) and by $\mathbf{X}_{h}=(-\partial h / \partial y, \partial h / \partial x)^{T}$ (the Hamiltonian vector field associated with the polynomial $h$ ). If $h \in \mathcal{P}_{r+|\mathbf{t}|}^{\mathbf{t}}$ then $\mathbf{X}_{h} \in \mathcal{Q}_{r}^{\mathbf{t}}$ where $|\mathbf{t}|=t_{1}+t_{2}$. Moreover, it is proved that every $\mathbf{F}_{j} \in \mathcal{Q}_{j}^{\mathbf{t}}$ can be expressed as

$$
\mathbf{F}_{j}=\mathbf{X}_{h_{j}}+\mu_{j} \mathbf{D}_{0}
$$

with $h_{j}=\left(\mathbf{D}_{0} \wedge \mathbf{F}_{j}\right) /(j+|\mathbf{t}|)$ and $\mu_{j}=\operatorname{div}\left(\mathbf{F}_{j}\right) /(j+|\mathbf{t}|)$, where $\mathbf{D}_{0} \wedge \mathbf{F}_{j}:=$ $t_{1} x Q_{j+t_{2}}-t_{2} y P_{j+t_{1}} \in \mathcal{P}_{j+|\mathbf{t}|}^{\mathbf{t}}$ and $\operatorname{div}\left(\mathbf{F}_{j}\right) \in \mathcal{P}_{j}^{\mathbf{t}}$ is the divergence of $\mathbf{F}_{j}$, see [4]. Our main result is the following.

Theorem 1.2. Let $\mathbf{F}$ be vector field with a monodromic nilpotent singularity at the origin then

(a) System $\dot{\mathbf{x}}=\mathbf{F}(\mathbf{x})$ can be transformed into

$$
\left(\begin{array}{c}
\dot{x} \\
\dot{y}
\end{array}\right)=\mathbf{F}_{n}+\left(\begin{array}{c}
F\left(x^{2}, y\right) \\
x G\left(x^{2}, y\right)
\end{array}\right)+\sum_{2 i>n} \alpha_{2 i} x^{2 i}\left(\begin{array}{c}
x \\
(n+1) y
\end{array}\right),
$$

where

$$
\mathbf{F}_{n}=\left(\begin{array}{c}
y \\
-(n+1) x^{2 n+1}
\end{array}\right)+d x^{n}\left(\begin{array}{c}
x \\
(n+1) y
\end{array}\right),
$$

i.e. $\mathbf{F}_{n}=\mathbf{X}_{h}+\mu \mathbf{D}_{0}$, where $h=-\frac{1}{2}\left(y^{2}+x^{2 n+2}\right), \mu=d x^{n}, \mathbf{D}_{0}=$ $(x,(n+1) y)^{T}$ and $\left(F\left(x^{2}, y\right), x G\left(x^{2}, y\right)\right)^{T}$ is a vector field sum of quasihomogeneous vector fields of type $\mathbf{t}$ and degree greater than $n$ where $n-1$ is the Andreev number, see [19].

(b) System (1.4) is a center if and only if $\alpha_{2 i}=0$ for all $2 i>n$ and the origin of $\dot{\mathbf{x}}=\mathbf{F}_{n}(\mathbf{x})$ is a center.

The proof of Theorem 1.2 is given in Sect. 2. In the particular case, $n$ even and $d=0$ this theorem was proved in [10]; nevertheless, we give a exhaustive proof for completeness.

The normal form (1.4) allows to characterize the nilpotent centers in a way more effective that transforming the system into the Bogdanov-Takens normal form (1.2). This is due to the fact that the normal form (1.4) is less elaborated than normal form (1.2) and less terms of the original system must be canceled to arrive to the normal form (1.4). Therefore, the procedure derived from the normal form (1.4) is more effective.

These constants $\alpha_{2 i}$ give the center conditions, and their vanish is a necessary condition to have a center. Therefore, Theorem 1.2 provides an effective algorithm for computing center conditions for general nilpotent systems. Notice that the algorithm is efficient because only use the change of variables necessary to achieve the reduced orbital normal form (1.4). This 
fact allows to calculate more Lyapunov constants than by the classical algorithm that includes all change of variables, as it is shown in the following proofs. Moreover, the constants $\alpha_{2 i}$ that appear in (1.4) give the conditions of orbital reversibility, that is, system $\dot{\mathbf{x}}=\mathbf{F}(\mathbf{x})$ of Theorem 1.2 is orbital reversible if, and only if, $\alpha_{2 i}=0$ for all $i \geq 0$ in the $\operatorname{system}(1.4)$, see $[1,22]$.

\section{Proof of Theorem 1.2}

The next result characterizes the form of the first quasi-homogeneous component of a vector field with an monodromic nilpotent singularity.

To prove the result, we first analyze the normal preform which sets the Newton diagram of the field and, therefore, the type and the degree of the quasi-homogeneous leading term of the vector field that we want to study. The next result establishes the normal preform of the quasi-homogeneous leading term.

Proposition 2.3 [8, Proposition 2.5]. Consider $\dot{\mathbf{x}}=\mathbf{F}(\mathbf{x})$ a nilpotent vector field with $\mathbf{0}$ an isolated singular point. There exists $n \in \mathbb{N}$, a polynomial change $\Phi$ and a type $\mathbf{t}$ such that $\tilde{\mathbf{F}}:=\Phi_{*} \mathbf{F}=\tilde{\mathbf{F}}_{r}+\cdots, \tilde{\mathbf{F}}_{r} \in \mathcal{Q}_{r}^{t}$, with $\cdots$ the quasi-homogeneous terms of type $\mathbf{t}$ and degree greater than $r$, where $\tilde{\mathbf{F}}_{r}$ satisfies one of the following two conditions:

(A) $\tilde{\mathbf{F}}_{r}=\left(y, b x^{n} y\right)^{T} \in \mathcal{Q}_{n}^{\mathbf{t}}, b \in \mathbb{R}, b \neq 0$, i.e., $\mathbf{t}=(1, n+1)$, and $r=n$. In this case $\mathbf{0}$ is not an isolated singular point of $\dot{\mathbf{x}}=\tilde{\mathbf{F}}_{r}(\mathbf{x})$.

(B) $\mathbf{0}$ is an isolated singular point of $\dot{\mathbf{x}}=\tilde{\mathbf{F}}_{r}(\mathbf{x})$ and there exist $\Psi_{0} \in \mathcal{Q}_{0}^{\mathbf{t}}$, with $\operatorname{det}\left(D \Psi_{0}(\mathbf{0})\right) \neq 0$ such that $\mathbf{G}_{r}=\left(\Psi_{0}\right)_{*} \tilde{\mathbf{F}}_{r}$ is one of the following vector fields.

$$
\begin{aligned}
& \mathbf{G}_{r}=\left(\begin{array}{c}
y \\
x^{2 n}
\end{array}\right) \in \mathcal{Q}_{2 n-1}^{(2,2 n+1)}, \text { i.e., } \mathbf{t}=(2,2 n+1) \text { and } r=2 n-1 . \\
& \mathbf{G}_{r}=\left(\begin{array}{c}
y \\
-(n+1) x^{2 n+1}
\end{array}\right)+d x^{n}\left(\begin{array}{c}
x \\
(n+1) y
\end{array}\right) \in \mathcal{Q}_{n}^{(1, n+1)}, d \in \mathbb{R}, \\
& \text { i.e., } \mathbf{t}=(1, n+1) \text { and } r=n . \\
& \mathbf{G}_{r}=\left(\begin{array}{c}
y \\
0
\end{array}\right)+d x^{n}\left(\begin{array}{c}
x \\
(n+1) y
\end{array}\right) \in \mathcal{Q}_{n}^{(1, n+1)}, d \in \mathbb{R}, d \neq 0, \text { i.e., } \\
& \mathbf{t}=(1, n+1) \text { and } r=n . \\
& \mathbf{G}_{r}=\left(\begin{array}{c}
y \\
(n+1) x^{2 n+1}
\end{array}\right)+d x^{n}\left(\begin{array}{c}
x \\
(n+1) y
\end{array}\right) \in \mathcal{Q}_{n}^{(1, n+1)}, d \in \mathbb{R}, \\
& d \neq 1, \text { i.e., } \mathbf{t}=(1, n+1) \text { and } r=n .
\end{aligned}
$$

Proposition 2.4. Consider $\dot{\mathbf{x}}=\mathbf{F}(\mathbf{x})$ a nilpotent vector field where the origin is monodromic then there exists $n \in \mathbb{N}$, a polynomial change $\Phi$ and a type $\mathbf{t}=(1, n+1)$ such that this system is conjugate to $\dot{\mathbf{x}}=\mathbf{F}_{n}(\mathbf{x})+\cdots$, where $\mathbf{F}_{n}$ is given in (1.5) and $\cdots$ means higher terms quasi-homogeneous respect to the type $\mathbf{t}=(1, n+1)$.

Proof. Applying Proposition 2.3, the unique $\mathbf{G}_{r}$ which is monodromic corresponds to case B2).

Therefore, it is enough to study the systems $\dot{\mathbf{x}}=\mathbf{F}_{n}+\cdots$, where $\mathbf{F}_{n}$ is given in (1.5) 
Theorem 2.5. If the origin of $\dot{\mathbf{x}}=\mathbf{F}_{n}+\cdots$ where $\mathbf{F}_{n}$ is given in (1.5) is a center then the origin of system $\dot{\mathbf{x}}=\mathbf{F}_{n}(\mathbf{x})$ is also a center.

Proof. Indeed, the result is a particular case of [3, Theorem 5]

From Theorem 2.5, to study the problem of center for nilpotent systems it is necessary to study when the origin of $\dot{\mathbf{x}}=\mathbf{F}_{n}(\mathbf{x}), \mathbf{F}_{n}$ is given in (1.5), is a center.

Proposition 2.6. Let $\mathbf{F}_{n}$ the quasi-homogeneous vector field (1.5). The origin of system $\dot{\mathbf{x}}=\mathbf{F}_{n}(\mathbf{x})$ is a center if, and only if one of following conditions is satisfied (a) $n$ is odd, or (b) $n$ is even and $d=0$.

Proof. We will use generalized polar coordinates: $x=\rho \operatorname{Cs}(\theta), y=\rho^{n+1} \operatorname{Sn}(\theta)$, where $(\operatorname{Cs}(\theta), \operatorname{Sn}(\theta))^{T}$ is the solution of the initial value problem $\dot{\mathbf{x}}=\mathbf{X}_{g}(\mathbf{x})$, $\mathbf{x}(0)=(1,0)^{T}$, being $g=x^{2 n+2}+y^{2}$. It is well known that these functions are periodic functions, and they satisfy $\mathrm{Cs}^{2 n+2}(\theta)+\operatorname{Sn}^{2}(\theta)=1$.

Observe that $\mathbf{F}_{n}=\dot{\mathbf{x}}=\frac{1}{\rho} \mathbf{D}_{0} \dot{\rho}+\frac{1}{\rho^{n}} \mathbf{X}_{g} \dot{\theta}$. Then taking in account that $\mathbf{F}_{n}=-\frac{1}{2} \mathbf{X}_{g}+\mathrm{d} x^{n} \mathbf{D}_{0}$, we get $\mathbf{F}_{n} \wedge \mathbf{X}_{g}=\frac{1}{\rho} \mathbf{D}_{0} \wedge \mathbf{X}_{g} \dot{\rho}, \mathbf{D}_{0} \wedge \mathbf{F}_{n}=\frac{1}{\rho^{n}} \mathbf{D}_{0} \wedge \mathbf{X}_{g} \dot{\theta}$. As $\mathbf{F}_{n} \wedge \mathbf{X}_{g}=d x^{n} \mathbf{D}_{0} \wedge \mathbf{X}_{g}, \mathbf{D}_{0} \wedge \mathbf{F}_{n}=-\frac{1}{2} \mathbf{D}_{0} \wedge \mathbf{X}_{g}$ and $\mathbf{D}_{0} \wedge \mathbf{X}_{g}=2(n+$ 1) $\left(x^{2 n+2}+y^{2}\right)=2(n+1) \rho^{2 n+2}\left(\mathrm{Cs}^{2 n+2}(\theta)+\operatorname{Sn}^{2}(\theta)\right)=2(n+1) \rho^{2 n+2}$, after the time-rescaling $\mathrm{d} T=-\frac{\rho^{n}}{2} \mathrm{~d} t$, we get

$$
\begin{aligned}
& \frac{\mathrm{d} \rho}{\mathrm{d} T}=-2 d \mathrm{Cs}^{n}(\theta) \rho, \\
& \frac{\mathrm{d} \theta}{\mathrm{d} T}=1 .
\end{aligned}
$$

Consequently there are no equilibria on the axis $\rho=0$, and the origin of system $\dot{\mathbf{x}}=\mathbf{F}_{n}(\mathbf{x})$ is monodromic. If $n$ is odd the system $\dot{\mathbf{x}}=\mathbf{F}_{n}(\mathbf{x})$ is invariant respect to $(x, y, t) \rightarrow(-x, y,-t)$; therefore, the origin is a center. It is case (a). If $n$ is even and $d=0, \mathbf{F}_{n}$ is a Hamiltonian vector field; therefore, the origin is a center. It is case (b). If $n$ is even and $d \neq 0$, the origin of system $\dot{\mathbf{x}}=\mathbf{F}_{n}(\mathbf{x})$ is a focus and its stability can be easily deduced from system (2.6).

Therefore, for studying the center problem for nilpotent singularities, it is enough to analyze the center problem for systems

$$
\dot{\mathbf{x}}=\mathbf{F}(\mathbf{x}):=\mathbf{F}_{n}(\mathbf{x})+\cdots,
$$

where $\mathbf{F}_{n}$ is given in (1.5), $\cdots$ means higher terms quasi-homogeneous respect to the type $\mathbf{t}=(1, n+1)$ and the origin of $\dot{\mathbf{x}}=\mathbf{F}_{n}(\mathbf{x})$ is a center, i.e. $n$ is odd or $n$ is even and $d=0$.

\subsection{Orbital Normal Form}

Next result provides the orbital normal form of system (2.7).

Theorem 2.7. Let $\mathbf{F}$ the vector field given in (2.7), then $\mathbf{F}$ is orbital equivalent to $\mathbf{G}=\mathbf{F}_{n}+\sum_{k>0} \nu_{k+n} \mathbf{D}_{0}$, where $\nu_{k+n} \in \operatorname{Cor}\left(\ell_{k+n}\right)$ (complementary subspace to Range $\left(\ell_{k+n}\right)$ ) and $\ell_{k+n}$ is the Lie-derivative operator of $\mathbf{F}_{n}$, i.e.,

$$
\begin{aligned}
\ell_{k+n}: \mathcal{P}_{k}^{\mathbf{t}} & \longrightarrow \mathcal{P}_{k+n}^{\mathbf{t}} \\
\mu_{k} & \longrightarrow \nabla \mu_{k} \cdot \mathbf{F}_{n} .
\end{aligned}
$$


Proof. By [8, Theorem 3.18], given $\mathbf{F}=\sum_{j \geq n} \mathbf{F}_{j}$ with $\mathbf{F}_{j} \in \mathcal{Q}_{j}^{\mathbf{t}}$. If Ker $\left(\ell_{n+k+|\mathbf{t}|}^{c}\right)=\{0\}$ for all $k \in \mathbb{N}$, then $\mathbf{F}$ is orbitally equivalent to

$$
\mathbf{G}=\mathbf{F}_{n}+\sum_{k>0} \mathbf{G}_{k+n}, \text { with } \mathbf{G}_{k+n}=\mathbf{X}_{g_{k+n+|\mathbf{t}|}}+\eta_{k+n} \mathbf{D}_{0} \in \mathcal{Q}_{k+n}^{\mathbf{t}},
$$

where $g_{k+n+|\mathbf{t}|} \in \operatorname{Cor}\left(\ell_{n+k+|\mathbf{t}|}^{c}\right)$ and $\eta_{k+n} \in \operatorname{Cor}\left(\ell_{k+n}\right), \ell_{k+n}$ is the Liederivative operator of $\mathbf{F}_{n}$,

$$
\begin{aligned}
\ell_{n+k+|\mathbf{t}|}^{\mathrm{c}} & : \Delta_{k+|\mathbf{t}|} \longrightarrow \Delta_{n+k+|\mathbf{t}|} \\
& g \rightarrow \operatorname{Proy}_{\Delta_{n+k+|\mathbf{t}|}}\left(\nabla g \cdot\left(\mathbf{F}_{n}-\frac{n+|\mathbf{t}|}{n+k+|\mathbf{t}|} \mu \mathbf{D}_{0}\right)\right)
\end{aligned}
$$

$\mu=\frac{1}{n+|\mathbf{t}|} \operatorname{div}\left(\mathbf{F}_{n}\right)$ and $\Delta_{k+|\mathbf{t}|}$, is a complementary subspace to $h \mathcal{P}_{k-n}^{\mathbf{t}}$ in $\mathcal{P}_{k+|\mathbf{t}|}^{\mathbf{t}}$, where $h=\frac{1}{n+|\mathbf{t}|} \mathbf{D}_{0} \wedge \mathbf{F}_{n}$.

It is sufficient to prove that $\operatorname{Ker}\left(\ell_{k+2(n+1)}^{\mathrm{c}}\right)=\{0\}$ and $\operatorname{Cor}\left(\ell_{k+2(n+1)}^{\mathrm{c}}\right)$ $=\{0\}$ for all $k \in \mathbb{N}$.

Let us take $p \in \Delta_{k+n+2}$, where $\Delta_{k+n+2}$ is a complementary subspace to $h \mathcal{P}_{k-n}^{\mathbf{t}}$ in $\mathcal{P}_{k+n+2}^{\mathbf{t}}$. Then we can write

$$
p(x, y)=\alpha_{0} x^{k+n+2}+\alpha_{1} x^{k+1} y .
$$

Consider $\mathbf{G}_{n}=\mathbf{F}_{n}-\frac{2(n+1)}{k+2(n+1)} \mathrm{d} x^{n} \mathbf{D}_{0}$, then

$$
\begin{aligned}
\nabla p(x, y) \cdot \mathbf{G}_{n}= & (k+n+2) \alpha_{0} x^{k+n+1} \nabla x \cdot \mathbf{G}_{n} \\
& +(k+1) \alpha_{1} x^{k} y \nabla x \cdot \mathbf{G}_{n}+\alpha_{1} x^{k+1} \nabla y \cdot \mathbf{G}_{n} .
\end{aligned}
$$

Taking into account that

$$
\begin{aligned}
\nabla x \cdot \mathbf{G}_{n} & =y+\frac{k}{k+2(n+1)} \mathrm{d} x^{n+1}, \\
\nabla y \cdot \mathbf{G}_{n} & =-(n+1) x^{2 n+1}+\frac{(n+1) k}{k+2(n+1)} \mathrm{d} x^{n} y, \\
y^{2} & =-x^{2 n+2}-2 h,
\end{aligned}
$$

we have that

$$
\begin{aligned}
\nabla p(x, y) \cdot \mathbf{G}_{n}= & \frac{k+n+2}{k+2(n+1)}\left[d k \alpha_{0}-(k+2(n+1)) \alpha_{1}\right] x^{k+2(n+1)} \\
& +\frac{k+n+2}{k+2(n+1)}\left[(k+2(n+1)) \alpha_{0}+k d \alpha_{1}\right] x^{k+n+1} y \\
& -2(k+1) \alpha_{1} x^{k} h .
\end{aligned}
$$

In this way,

$$
\begin{gathered}
\ell_{k+2(n+1)}^{\mathrm{c}}(p)=\frac{k+n+2}{k+2(n+1)} x^{k+n+1}\left[\left[d k \alpha_{0}-(k+2(n+1)) \alpha_{1}\right] x^{n+1}\right. \\
\left.+\left[(k+2(n+1)) \alpha_{0}+k d \alpha_{1}\right] y\right] .
\end{gathered}
$$

Hence, choosing the bases $\Delta_{k+n+2}=\operatorname{span}\left\{x^{k+n+2}, x^{k+1} y\right\}$ and $\Delta_{k+2(n+1)}=$ $\operatorname{span}\left\{x^{k+2(n+1)}, x^{k+n+1} y\right\}$. Notice that $\operatorname{dim}\left(\Delta_{k+n+2}\right)=\operatorname{dim}\left(\Delta_{k+2(n+1)}\right)$, we obtain that the matrix of the operator $\ell_{k+2(n+1)}^{\mathrm{c}}$ is

$$
\left(\begin{array}{cc}
d k & -(k+2(n+1)) \\
k+2(n+1) & d k
\end{array}\right)
$$


from the structure of the above matrix, the proof follows.

2.1.1. Computation of Cor $\left(\ell_{k+n}\right)$. In this subsection, we calculate the coranges that are necessary for computing a orbital normal for of system (2.7). For that, we need the following results:

Lemma 2.8 [8, Lemma 3.24]. Consider $p \in \mathcal{P}_{k}^{\mathbf{t}}, \mathbf{F}_{n}=\mathbf{X}_{h}+\mu \mathbf{D}_{0} \in \mathcal{Q}_{n}^{\mathbf{t}}$ irreducible and $f \in \mathcal{P}_{s}^{\mathbf{t}}$ an irreducible quasi-homogeneous invariant curve of $\mathbf{F}_{n}$ with $K_{n}$ its cofactor. If $\ell_{n+k}(p) \in\left\langle f^{m}\right\rangle$ and $\mathbf{F}_{n}+\frac{j K_{n}}{k-j s} \mathbf{D}_{0}$ is irreducible for $j=1, \ldots, m-1$ then $p \in\left\langle f^{m}\right\rangle$.

Definition 2.9. A vector field $\mathbf{F}$ is reducible there exist a scalar function $f$ and a vector field $\mathbf{G}$ such that $\mathbf{F}=f \mathbf{G}$.

Proposition 2.10 [2, Theorem 4.13]. The irreducible quasi-homogeneous vector field $\mathbf{F}_{n}=\mathbf{X}_{h}+\mu \mathbf{D}_{0} \in \mathcal{Q}_{n}^{\mathbf{t}}$ has a polynomial first integral if, and only if, there exists $\frac{r}{s} \in \mathbb{Q}_{+}$(positive rational numbers) such that $\mathbf{F}_{n}+\frac{r}{s} \mu \mathbf{D}_{0}$ is reducible.

Lemma 2.11. Let the vector field $\mathbf{F}_{n}=\mathbf{X}_{h}+\mu \mathbf{D}_{0}$ given in (1.5), then the vector field $\mathbf{F}_{n}+\frac{r}{s} \mu \mathbf{D}_{0}$ is not reducible for all $\frac{r}{s}$ positive rational number.

Proof. If $d=0$ then $\mu=0$ and $\mathbf{F}_{n}=\mathbf{X}_{h}+\frac{r}{s} \mu \mathbf{D}_{0}=\mathbf{X}_{h}$ is not reducible.

If $d \neq 0$, by Proposition 2.10, the vector field $\mathbf{F}_{n}+\frac{r}{s} \mu \mathbf{D}_{0}$ is not reducible for all $\frac{r}{s}$ positive rational number since by Proposition $2.12 \mathbf{F}_{n}$ is not integrable.

Proposition 2.12. The vector field $\mathbf{F}_{n}=\mathbf{X}_{h}+\mu \mathbf{D}_{0}$ given in (1.5) is analytic integrable if, and only if, $d=0$. Moreover, a primitive first integral is $h$.

Proof. As $\mathbf{F}_{n}$ is a quasi-homogeneous vector field, if $\mathbf{F}_{n}$ is integrable there exists a quasi-homogeneous first integral. This first integral is product of invariant curves of $\mathbf{F}_{n}$. The only irreducible quasi-homogeneous invariant curve of $\mathbf{F}_{n}$ is $h$. Therefore, if $\mathbf{F}_{n}$ is integrable there exists a first integral $I=h^{l}, l \in \mathbb{N}$. But

$$
\nabla h^{l} \cdot \mathbf{F}_{n}=\nabla h^{l} \cdot\left(\mathrm{d} x^{n} \mathbf{D}_{0}\right)=(2 n+2) l \mathrm{~d} x^{n} h^{l} .
$$

So $\nabla h^{l} \cdot \mathbf{F}_{n}=0$, if and only if $d=0$. In this case, a primitive first integral is $h$.

Corollary 2.13. Let the vector field $\mathbf{F}_{n}=\mathbf{X}_{h}+\mu \mathbf{D}_{0}$ given in (1.5) and $\ell_{k+n}$ the Lie-derivative operator of $\mathbf{F}_{n}$ then

$$
\operatorname{Ker}\left(\ell_{k+n}\right)= \begin{cases}\operatorname{span}\left\{h^{l}\right\} & \text { if } d=0, k=2 l(n+1) \\ \{0\} & \text { if } d \neq 0 \text { or } \bmod (k, 2(n+1)) \neq 0\end{cases}
$$

Proof. It is a direct application of Proposition 2.12.

Next result provides us a cyclicity property for the co-range of the Lie operator of $\mathbf{F}_{n}$. Hence, it is enough for computing the formal orbital normal form to calculate a finite number of them. 
Proposition 2.14. Let $\mathbf{F}_{n}=\mathbf{X}_{h}+\mu \mathbf{D}_{0}$ given in (1.5) and $k \in \mathbb{N}$. It is possible to choose a complementary subspace to Range $\left(\ell_{k+n}\right)$ such that

$$
\operatorname{Cor}\left(\ell_{k+n}\right)=h^{l} \operatorname{Cor}\left(\ell_{i}^{(l)}\right) \text {, }
$$

where Cor $\left(\ell_{i}^{(l)}\right)$ is a complementary subspace to Range $\left(\ell_{i}^{(l)}\right)$ and the linear operator $\ell_{i}^{(l)}$ is defined by

$$
\begin{aligned}
\ell_{i}^{(l)} & : \mathcal{P}_{i-n}^{\mathbf{t}} \\
& \longrightarrow \mathcal{P}_{i}^{\mathbf{t}} \\
& \mu_{i-n} \rightarrow \nabla \mu_{i-n} \cdot\left(\mathbf{F}_{n}+\frac{2(n+1) l}{i} \mu \mathbf{D}_{0}\right)
\end{aligned}
$$

being $i=\left\{\begin{array}{ll}i_{0}+2(n+1) & \text { if } 0 \leq i_{0} \leq n \\ i_{0} & \text { otherwise }\end{array}\right.$, where $i_{0}:=\bmod (k+n, 2(n+1))$ and $l:=\frac{k+n-i}{2(n+1)}$.

Proof. We will prove that $h^{l} \operatorname{Cor}\left(\ell_{i}^{(l)}\right) \subset \operatorname{Cor}\left(\ell_{k+n}\right)$ or equivalently that $h^{l} \operatorname{Cor}\left(\ell_{i}^{(l)}\right) \cap \operatorname{Range}\left(\ell_{k+n}\right)=\{0\}$ by reductio ad absurdum. Given $p \in$ Cor $\left(\ell_{i}^{(l)}\right) \backslash\{0\}$ such that $p h^{l} \in$ Range $\left(\ell_{k+n}\right)$, then there exists $q \in \mathcal{P}_{k}^{\mathbf{t}} \backslash\{0\}$, such that $\ell_{k+n}(q)=p h^{l}$. Applying Lemmas 2.11 and 2.8 in the case $f=h$, $K_{n}=2(n+1) \mu$, we have that there exits $\tilde{q} \in \mathcal{P}_{i-n}^{\mathbf{t}} \backslash\{0\}$ such that $q=\tilde{q} h^{l}$ and consequently

$$
\begin{aligned}
p h^{l}= & \nabla q \cdot \mathbf{F}_{n}=\nabla \tilde{q} h^{l} \cdot \mathbf{F}_{n}=h^{l} \nabla \tilde{q} \cdot \mathbf{F}_{n}+l q h^{l-1} \nabla h \cdot \mathbf{F}_{n}=h^{l} \nabla \tilde{q} \cdot \mathbf{F}_{n} \\
& +2(n+1) l q \mu h^{l}=h^{l} \nabla \tilde{q} \cdot\left(\mathbf{F}_{n}+\frac{2(n+1) l}{i} \mu \mathbf{D}_{0}\right) .
\end{aligned}
$$

Hence, $p=\nabla \tilde{q} \cdot\left(\mathbf{F}_{n}+\frac{2(n+1) l}{i} \mu \mathbf{D}_{0}\right)$, that is, $p \in \operatorname{Range}\left(\ell_{i}^{(l)}\right) \cap \operatorname{Cor}\left(\ell_{i}^{(l)}\right)$ which gives a contradiction.

We have shown that $h^{l} \operatorname{Cor}\left(\ell_{i}^{(l)}\right) \subset \operatorname{Cor}\left(\ell_{k+n}\right)$. Therefore, it is enough to prove that $\operatorname{dim}\left(h^{l} \operatorname{Cor}\left(\ell_{i}^{(l)}\right)\right)=\operatorname{dim}\left(\operatorname{Cor}\left(\ell_{k+n}\right)\right)$.

Since $\ell_{i}^{(l)}$ and $\ell_{k+n}$ are linear operators, we get

$$
\begin{aligned}
\operatorname{dim}\left(\operatorname{Cor}\left(\ell_{i}^{(l)}\right)\right) & =\operatorname{dim}\left(\mathcal{P}_{i}^{\mathbf{t}}\right)-\operatorname{dim}\left(\mathcal{P}_{i-n}^{\mathbf{t}}\right)+\operatorname{dim}\left(\operatorname{Ker}\left(\ell_{i}^{(l)}\right)\right) . \\
\operatorname{dim}\left(\operatorname{Cor}\left(\ell_{k+n}\right)\right) & =\operatorname{dim}\left(\mathcal{P}_{k+n}^{\mathbf{t}}\right)-\operatorname{dim}\left(\mathcal{P}_{k}^{\mathbf{t}}\right)+\operatorname{dim}\left(\operatorname{Ker}\left(\ell_{k+n}\right)\right) .
\end{aligned}
$$

If $d=0$ then $\mu=0$ and $\ell_{i}^{(l)}=\ell_{i}$, on the other hand, as $\bmod (k+n, n+|\mathbf{t}|)=$ $\bmod (i, n+|\mathbf{t}|)$ applying Corollary 2.13 we get $\operatorname{dim}\left(\operatorname{Ker}\left(\ell_{i}\right)\right)=\operatorname{dim}\left(\operatorname{Ker}\left(\ell_{k+n}\right)\right)$; therefore, we get $\operatorname{dim}\left(\operatorname{Ker}\left(\ell_{i}^{(l)}\right)\right)=\operatorname{dim}\left(\operatorname{Ker}\left(\ell_{k+n}\right)\right)$.

If $d \neq 0$ by Corollary $2.13 \operatorname{Ker}\left(\ell_{k+n}\right)=\{0\}$ for all $k \in \mathbb{N}$. Also $\operatorname{Ker}\left(\ell_{i}^{(l)}\right)=\{0\}$ since $i>n$, otherwise by Proposition 2.10 there exists $\frac{r}{s} \in \mathbb{Q}_{+}$such that $\mathbf{F}_{n}+\frac{2(n+1) l}{i} \mu \mathbf{D}_{0}+\frac{r}{s} \mu \mathbf{D}_{0}$ is reducible, i.e. $\mathbf{F}_{n}+\frac{\tilde{r}}{\tilde{s}} \mu \mathbf{D}_{0}$ is reducible for $\frac{\tilde{r}}{\tilde{s}}=\frac{2(n+1) l}{i}+\frac{r}{s}$ and by Lemma 2.11 this is a contradiction.

Therefore, $\operatorname{dim}\left(\operatorname{Ker}\left(\ell_{i}^{(l)}\right)\right)=\operatorname{dim}\left(\operatorname{Ker}\left(\ell_{k+n}\right)\right)$ and from $(2.8),(2.9)$, it is enough to prove that

$$
\operatorname{dim}\left(\mathcal{P}_{k+n}^{\mathbf{t}}\right)-\operatorname{dim}\left(\mathcal{P}_{k}^{\mathbf{t}}\right)=\operatorname{dim}\left(\mathcal{P}_{i}^{\mathbf{t}}\right)-\operatorname{dim}\left(\mathcal{P}_{i-n}^{\mathbf{t}}\right) .
$$


Taking into account that $\mathcal{P}_{i-n}^{\mathbf{t}} \neq\{0\}$ since $i-n>0$, and $h=\frac{1}{2}\left(y^{2}+\right.$ $\left.x^{2(n+1)}\right)$; therefore, $\operatorname{deg}(h)=2(n+1)$ and applying [4, Lemma 2.3] we obtain $\operatorname{dim}\left(\operatorname{Cor}\left(\ell_{i}^{(l)}\right)\right)=\operatorname{dim}\left(\operatorname{Cor}\left(\ell_{k+n}\right)\right)$ and this completes the proof.

The following result provides explicit calculate of Cor $\left(\ell_{k+n}\right)$.

Proposition 2.15. Consider $\mathbf{F}_{n}=\mathbf{X}_{h}+\mu \mathbf{D}_{0}$ given in (1.5), $k \in \mathbb{N}$. Then $a$ complementary subspace to Range $\left(\ell_{k+n}\right)$ is

$$
\operatorname{Cor}\left(\ell_{k+n}\right)= \begin{cases}\operatorname{span}\left\{x^{i_{0}} h^{l+1}\right\}, & \text { if } 0 \leq i_{0}<n, \\ \operatorname{span}\left\{x^{i_{0}} h^{l+1}\right\}, & \text { if } i_{0}=n, d=0, \\ \operatorname{span}\left\{x^{i_{0}} h^{l}\right\}, & \text { if } n<i_{0}<2 n+1, \\ \{0\}, & \text { otherwise, }\end{cases}
$$

where $i_{0}, l$ are defined in Proposition 2.14.

Proof. By Proposition 2.14 it is enough to calculate Cor $\left(\ell_{i}^{(l)}\right)$, where $i$ and $l$ is defined in the quoted Proposition. We consider different cases in function $i_{0}=\bmod (k+n, 2(n+1))$

- Case $0 \leq i_{0}<n$ then $i=i_{0}+2(n+1)$, and $2(n+1) \leq i<3 n+2$, then $\mathcal{P}_{i-n}^{\mathbf{t}}=\operatorname{span}\left\{x^{i-n}, x^{i-2 n-1} y\right\}$.

If $p \in \mathcal{P}_{i-n}^{\mathbf{t}}$ then $p(x, y)=\alpha_{0} x^{i-n}+\alpha_{1} x^{i-2 n-1} y, \alpha_{0}, \alpha_{1} \in \mathbb{R}$ and

$$
\begin{aligned}
\ell_{i}^{(l)}(p)= & \nabla p \cdot\left(\mathbf{F}_{n}+\frac{l(k+n)}{i} \mu \mathbf{D}_{0}\right)=\nabla p \cdot\left(\mathbf{X}_{h}+\frac{k+n}{i} d x^{n} \mathbf{D}_{0}\right) \\
= & (i-n)\left(\frac{k+n}{i} d \alpha_{0}-\alpha_{1}\right) x^{i}+(i-n) \\
& \left(\alpha_{0}+\frac{k+n}{i} d \alpha_{1}\right) x^{i-n-1} y-2(i-2 n-1) \alpha_{1} x^{i-2 n-i} h .
\end{aligned}
$$

Taking $\mathcal{P}_{i}^{\mathbf{t}}=\operatorname{span}\left\{x^{i}, x^{i-n-1} y, x^{i-2(n+1)} h\right\}$ results: Cor $\left(\ell_{i}^{(l)}\right)=\operatorname{span}$ $\left\{x^{i-2(n+1)} h\right\}=\operatorname{span}\left\{x^{i_{0}} h\right\}$. By applying Proposition 2.14 Cor $\left(\ell_{k+n}\right)=$ $h^{l} \operatorname{span}\left\{x^{i_{0}} h\right\}=\operatorname{span}\left\{x^{i_{0}} h^{l+1}\right\}$.

- Case $i_{0}=n$ then $i=i_{0}+2(n+1)=3 n+2$ and $\mathcal{P}_{i-n}^{\mathbf{t}}=\operatorname{span}$ $\left\{x^{2 n+2}, x^{n+1} y, h\right\}$.

If $p \in \mathcal{P}_{i-n}^{\mathbf{t}}$, then $p(x, y)=\alpha_{0} x^{2 n+2}+\alpha_{1} x^{n+1} y+\alpha_{2} h, \alpha_{0}, \alpha_{1}, \alpha_{2} \in \mathbb{R}$ and

$$
\begin{aligned}
\ell_{i}^{(l)}(p)= & \nabla p \cdot\left(\mathbf{F}_{n}+\frac{l(k+n)}{i} \mu \mathbf{D}_{0}\right)=\nabla p \cdot\left(\mathbf{X}_{h}+\frac{k+n}{i} d x^{n} \mathbf{D}_{0}\right) \\
= & 2(n+1)\left[\left(\frac{k+n}{3 n+2} d \alpha_{0}-\alpha_{1}\right) x^{3 n+2}\right. \\
& \left.+\left(\alpha_{0}+\frac{k+n}{3 n+2} d \alpha_{1}\right) x^{n+1} y+\left(-\alpha_{1}+\frac{k+n}{3 n+2} d \alpha_{2}\right) x^{n} h\right] .
\end{aligned}
$$

Taking $\mathcal{P}_{3 n+2}^{\mathbf{t}}=\operatorname{span}\left\{x^{3 n+2}, x^{2 n+1} y, x^{n} h\right\}$ results: Cor $\left(\ell_{i}^{(l)}\right)=\{0\}$ if $d \neq 0$ and Cor $\left(\ell_{i}^{(l)}\right)=\operatorname{span}\left\{x^{n} h\right\}$ if $d=0$. By applying Proposition 2.14 Cor $\left(\ell_{k+n}\right)=\{0\}$, if $d \neq 0$ and $\operatorname{Cor}\left(\ell_{k+n}\right)=h^{l} \operatorname{span}\left\{x^{n} h\right\}=$ $\operatorname{span}\left\{x^{n} h^{l+1}\right\}$ if $d=0$.

- Case $n<i_{0}<2 n+1$, then $i=i_{0}$ and $\mathcal{P}_{i-n}^{\mathbf{t}}=\operatorname{span}\left\{x^{i-n}\right\}$. 
If $p \in \mathcal{P}_{i-n}^{\mathbf{t}}$, then $p(x, y)=\alpha_{0} x^{i-n}, \alpha_{0} \in \mathbb{R}$ and

$$
\begin{aligned}
\ell_{i}^{(l)}(p) & =\nabla p \cdot\left(\mathbf{F}_{n}+\frac{l(k+n)}{i} \mu \mathbf{D}_{0}\right)=\nabla p \cdot\left(\mathbf{X}_{h}+\frac{k+n}{i} d x^{n} \mathbf{D}_{0}\right) \\
& =(i-n)\left[\frac{k+n}{i} d \alpha_{0} x^{i}+\alpha_{0} x^{i-n-1} y\right]
\end{aligned}
$$

Taking $\mathcal{P}_{i}^{\mathbf{t}}=\operatorname{span}\left\{x^{i}, x^{i-n-1} y\right\}$ results: $\operatorname{Cor}\left(\ell_{i}^{(l)}\right)=\operatorname{span}\left\{x^{i}\right\}$. By applying Proposition 2.14, Cor $\left(\ell_{k+n}\right)=h^{l} \operatorname{span}\left\{x^{i}\right\}=\operatorname{span}\left\{x^{i_{0}} h^{l}\right\}$.

- Case $i_{0}=2 n+1$ then $i=i_{0}$ and $\mathcal{P}_{i-n}^{\mathbf{t}}=\operatorname{span}\left\{x^{n+1}, y\right\}$.

If $p \in \mathcal{P}_{i-n}^{\mathbf{t}}$ then $p(x, y)=\alpha_{0} x^{n+1}+\alpha_{1} y, \alpha_{0}, \alpha_{1} \in \mathbb{R}$ and

$$
\begin{aligned}
\ell_{i}^{(l)}(p) & =\nabla p \cdot\left(\mathbf{F}_{n}+\frac{l(k+n)}{i} \mu \mathbf{D}_{0}\right)=\nabla p \cdot\left(\mathbf{X}_{h}+\frac{k+n}{i} \mathrm{~d} x^{n} \mathbf{D}_{0}\right) \\
& =(n+1)\left[\left(\frac{k+n}{2 n+1} \mathrm{~d} \alpha_{0}+\alpha_{1}\right) x^{2 n+1}+\left(\alpha_{0}+\frac{k+n}{2 n+1} \mathrm{~d} \alpha_{1}\right) x^{n} y\right]
\end{aligned}
$$

Taking $\mathcal{P}_{i}^{\mathbf{t}}=\operatorname{span}\left\{x^{2 n+1}, x^{n} y\right\}$ results: Cor $\left(\ell_{i}^{(l)}\right)=\{0\}$. By Proposition 2.14, Cor $\left(\ell_{k+n}\right)=\{0\}$.

In the next result, we give a more suitable expression of Cor $\left(\ell_{k+n}\right)$.

Corollary 2.16. Consider $\mathbf{F}_{n}=\mathbf{X}_{h}+\mu \mathbf{D}_{0}$ given in (1.5), $k \in \mathbb{N}$. Then $a$ complementary subspace to Range $\left(\ell_{k+n}\right)$ is

$$
\operatorname{Cor}\left(\ell_{k+n}\right)=\left\{\begin{array}{lcc}
\operatorname{span}\left\{x^{k+n}\right\}, & \text { if } & i_{0} \neq 2 n+1 \text { and } i_{0} \neq n, \\
\operatorname{span}\left\{x^{k+n}\right\}, & \text { if } & i_{0}=n \text { and } d=0, \\
\{0\}, & \text { otherwise }
\end{array}\right.
$$

where $i_{0}$ is defined in Proposition 2.14.

Proof. We prove the corollary by reduction to the absurd. Assume that $x^{k+n} \in$ Range $\left(\ell_{k+n}\right)$. For $j=0, \ldots,\left\lfloor\frac{k}{n+1}\right\rfloor$, we have that

$$
\begin{aligned}
\nabla x^{k-j(n+1)} y^{j} \cdot \mathbf{F}_{n}= & -j(n+1) x^{k+2 n+1-j(n+1)} y^{j-1} \\
& +\mathrm{d} k x^{k+n-j(n+1)} y^{j}+(k-j(n+1)) x^{k-1-j(n+1)} y^{j+1} .
\end{aligned}
$$

Hence, for $j=0$ we get $\nabla x^{k} \cdot \mathbf{F}_{n}=\mathrm{d} k x^{k+n}+k x^{k-1} y$; therefore, if $x^{k+n} \in$ Range $\left(\ell_{k+n}\right)$, then $x^{k-1} y \in \operatorname{Range}\left(\ell_{k+n}\right)$. In the same way for $j=1$, we will have that $x^{k-n-2} y^{2} \in$ Range $\left(\ell_{k+n}\right)$ and reasoning in the same way $x^{k+n-j(n+1)} y^{j} \in \operatorname{Range}\left(\ell_{k+n}\right)$ for $j=0, \ldots, l$ with $l:=\left\lfloor\frac{k+n}{2(n+1)}\right\rfloor$. Consequently, $x^{i} h^{l} \in$ Range $\left(\ell_{k+n}\right)$ with $i=k+n-2(n+1) l$ which is in contradiction with Proposition 2.15.

\subsection{Reduced Orbital Normal Form}

Now we define the vector spaces which are useful for finding a reduced orbital normal form taking into account the reversibility property.

$$
\begin{aligned}
& \widetilde{\mathcal{Q}}_{k}^{\mathbf{t}}=\left\{(P, Q)^{T} \in \mathcal{Q}_{k}^{\mathbf{t}}: P(-x, y)=P(x, y), Q(-x, y)=-Q(x, y)\right\}, \\
&\text { (vector field reversible to } x) \\
& \overline{\mathcal{Q}}_{k}^{\mathbf{t}}=\left\{(P, Q)^{T} \in \mathcal{Q}_{k}^{\mathbf{t}}: P(-x, y)=-P(x, y), Q(-x, y)=Q(x, y)\right\},
\end{aligned}
$$


(symmetric vector field)

$$
\begin{aligned}
& \widetilde{\mathcal{P}}_{k}^{\mathbf{t}}=\left\{\mu \in \mathcal{P}_{k}^{\mathbf{t}}: \mu(-x, y)=-\mu(x, y)\right\}, \text { (odd scalar function) } \\
& \overline{\mathcal{P}}_{k}^{\mathbf{t}}=\left\{\mu \in \mathcal{P}_{k}^{\mathbf{t}}: \mu(-x, y)=\mu(x, y)\right\} \text { (even scalar function) }
\end{aligned}
$$

$\widetilde{\mathcal{Q}}_{k}^{\mathrm{t}}, \overline{\mathcal{Q}}_{k}^{\mathrm{t}}$ are the vector field in $\mathcal{Q}_{k}^{\mathrm{t}}$ such that the differential systems associated to with these vector field are invariant to $(x, y, t) \rightarrow(-x, y,-t)$, or invariant to $(x, y, t) \rightarrow(-x, y, t)$, respectively. It is verified that $\mathcal{Q}_{k}^{\mathrm{t}}=\widetilde{\mathcal{Q}}_{k}^{\mathrm{t}} \oplus \overline{\mathcal{Q}}_{k}^{\mathrm{t}}$ and $\mathcal{P}_{k}^{\mathbf{t}}=\widetilde{\mathcal{P}}_{k}^{\mathbf{t}} \oplus \overline{\mathcal{P}}_{k}^{\mathbf{t}}$.

The vector field transformed of $\mathbf{F}$ by means of the change of variables with generators (spatial $\mathbf{U}$ and temporal $\mu$ ) is given by (see [26])

$$
\mathbf{U}_{* *}(\mu \mathbf{F}):=\mu \mathbf{F}+[\mu \mathbf{F}, \mathbf{U}]+\frac{1}{2 !}[[\mu \mathbf{F}, \mathbf{U}], \mathbf{U}]+\frac{1}{3 !}[[[\mu \mathbf{F}, \mathbf{U}], \mathbf{U}], \mathbf{U}]+\cdots .
$$

Next result provides a simplified orbital normal form which uses reduced changes of variables and time reparametrization that are convenient for calculating necessary conditions of centers for systems with first quasihomogeneous component $\mathbf{F}_{n}$ given in (1.5) such that the origin of $\dot{\mathbf{x}}=\mathbf{F}_{n}(\mathbf{x})$ is a center.

Proof statement (a) of Theorem 1.2. By [14, Proposition 12-15] a formally orbital equivalent normal form of system $\dot{\mathbf{x}}=\mathbf{F}(\mathbf{x})$ is $\dot{\mathbf{x}}=\mathbf{G}(\mathbf{x}):=\mathbf{F}_{n}+$ $\sum_{k>0} \mathbf{G}_{k+n}$, with $\mathbf{G}_{k+n} \in \operatorname{Cor}\left(\mathcal{L}_{k+n}\right)$ (complementary space to the range of $\left.\mathcal{L}_{k+n}\right)$, being the homological operator

$$
\begin{aligned}
\mathcal{L}_{k+n}: \mathcal{Q}_{k}^{\mathbf{t}} \times \underset{\operatorname{Cor}\left(\ell_{k}\right)}{ } \rightarrow & \mathcal{Q}_{k+n}^{\mathbf{t}} \\
\left(\mathbf{U}_{k}, \mu_{k}\right) & -\left[\mathbf{F}_{n}, \mathbf{U}_{k}\right]-\mu_{k} \mathbf{F}_{n} .
\end{aligned}
$$

Moreover, from Theorem 2.7, $\mathbf{G}=\mathbf{F}_{n}+\sum_{k>0} \nu_{k+n} \mathbf{D}_{0}$ with $\nu_{k+n} \in \operatorname{Cor}\left(\ell_{k+n}\right)$ (complementary space to the range of $\left.\ell_{i}\right)$; therefore, $\operatorname{Cor}\left(\mathcal{L}_{k+n}\right)=\operatorname{Cor}\left(\ell_{k+n}\right) \mathbf{D}_{0}$. By Corollary 2.16, we have that

$\nu_{k+n}=\left\{\begin{array}{c}\beta_{k+n} x^{k+n} \text { if }\left\{\begin{array}{c}\bmod (k+n, 2(n+1)) \neq n \text { and } \bmod (k+n, 2(n+1)) \neq 2 n+1 \\ \text { or } \\ 0\end{array} \quad \begin{array}{c}\bmod (k+n, 2(n+1))=n \\ \text { otherwise }\end{array}\right.\end{array}\right.$

It is possible to choose a complementary subspace to the range of $\ell_{k+n}$, $\operatorname{Cor}\left(\ell_{k+n}\right)$, for all $k$, of the form $\operatorname{Cor}\left(\ell_{k+n}\right)=\widehat{\operatorname{Cor}\left(\ell_{k+n}\right)} \oplus \overline{\operatorname{Cor}\left(\ell_{k+n}\right)}$, where $\operatorname{Cor}\left(\ell_{k+n}\right)=\operatorname{Cor}\left(\ell_{k+n}\right) \cap \widetilde{\mathcal{P}}_{k+n}^{\mathbf{t}}$ and $\overline{\operatorname{Cor}\left(\ell_{k+n}\right)}=\operatorname{Cor}\left(\ell_{k+n}\right) \cap \overline{\mathcal{P}}_{k+n}^{\mathbf{t}}$. Therefore, $\mathcal{Q}_{k}^{\mathbf{t}} \times \operatorname{Cor}\left(\ell_{k}\right)=\widetilde{\mathcal{Q}}_{k}^{\mathbf{t}} \times \widetilde{\operatorname{Cor}\left(\ell_{k}\right)} \bigoplus \overline{\mathcal{Q}}_{k}^{\mathrm{t}} \times \overline{\operatorname{Cor}\left(\ell_{k}\right)}$.

With this choice, we consider again the operator $\mathcal{L}_{k+n}: \widetilde{\mathcal{Q}}_{k}^{\mathrm{t}} \times \widetilde{\operatorname{Cor}\left(\ell_{k}\right)} \bigoplus$ $\overline{\mathcal{Q}}_{k}^{\mathrm{t}} \times \overline{\operatorname{Cor}\left(\ell_{k}\right)} \rightarrow \overline{\mathcal{Q}}_{k+n}^{\mathrm{t}} \oplus \widetilde{\mathcal{Q}}_{k+n}^{\mathrm{t}}$ whose matrix can be expressed as

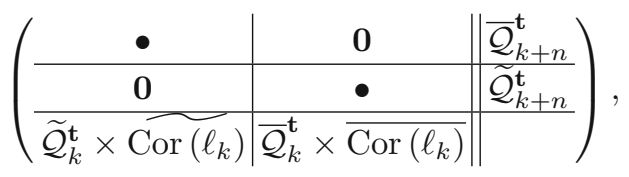


where $\bullet$ means a non-null submatrix. As the origin of $\dot{\mathbf{x}}=\mathbf{F}_{n}(\mathbf{x})$ is a center, by Proposition 2.6, $n$ is odd or $n$ is even and $d=0$ in both cases $\mathbf{F}_{n} \in \widetilde{\mathcal{Q}}_{n}^{\mathbf{t}}$ and hence the following properties are verified (see [15, Lemma 2.3, Lemma 2.7]: $\left[\mathbf{F}_{n}, \widetilde{\mathbf{U}}_{k}\right] \in \overline{\mathcal{Q}}_{k+n}^{\mathrm{t}},\left[\mathbf{F}_{n}, \overline{\mathbf{U}}_{k}\right] \in \widetilde{\mathcal{Q}}_{k+n}^{\mathrm{t}}$ and if $\widetilde{\mu}_{k} \in \widehat{\operatorname{Cor}\left(\ell_{k}\right)}, \bar{\mu}_{k} \in \overline{\operatorname{Cor}\left(\ell_{k}\right)}$, then $\widetilde{\mu}_{k} \mathbf{F}_{n} \in \overline{\mathcal{Q}}_{k+n}^{\mathbf{t}}$ and $\bar{\mu}_{k} \mathbf{F}_{n} \in \widetilde{\mathcal{Q}}_{k+n}^{\mathrm{t}}$.

Therefore, if we define the reduced operator

$$
\begin{aligned}
& \overline{\mathcal{L}}_{k+n}: \widetilde{\mathcal{Q}}_{k}^{\mathbf{t}} \times \widetilde{\operatorname{Cor}\left(\ell_{k}\right)} \rightarrow \overline{\mathcal{Q}}_{k+n}^{\mathbf{t}} \\
& \left(\tilde{\mathbf{U}}_{k}, \tilde{\mu}_{k}\right)-\left[\mathbf{F}_{n}, \tilde{\mathbf{U}}_{k}\right]-\tilde{\mu}_{k} \mathbf{F}_{n}
\end{aligned}
$$

the matrix of the reduced operator $\overline{\mathcal{L}}_{k+n}$ is a submatrix of the matrix of $\mathcal{L}_{k+n}$ and the formally orbital equivalent normal form using reduced spatial generators $\widetilde{\mathbf{U}}_{k} \in \widetilde{\mathcal{Q}}_{k}^{\mathbf{t}}$ and reduced temporal generators $\widetilde{\mu}_{k} \in \widetilde{\operatorname{Cor}\left(\ell_{k}\right)}$ is given by

$$
\widetilde{\mathbf{U}}_{* *}(\widetilde{\mu} \mathbf{F})=\mathbf{F}_{n}+\left(\begin{array}{c}
F\left(x^{2}, y\right) \\
x G\left(x^{2}, y\right)
\end{array}\right)+\sum_{2 i>n} \alpha_{2 i} x^{2 i} \mathbf{D}_{0}
$$

and the result follows.

Next result characterizes the centers of system (2.7).

Proof statement (b) of Theorem 1.2. By item (a) of Theorem 1.2 the origin of system $\dot{\mathbf{x}}=\mathbf{F}(\mathbf{x})$ is a center if, and only if, the origin of system $\dot{\mathbf{x}}=$ $\widetilde{\mathbf{U}}_{* *}(\tilde{\mu} \mathbf{F})(\mathbf{x})$ defined in $(1.4)$ is a center. If $\alpha_{2 i}=0$ for all $2 i>n$ then the system $\dot{\mathbf{x}}=\widetilde{\mathbf{U}}_{* *}(\tilde{\mu} \mathbf{F})(\mathbf{x})$ is reversible to $x$ and its origin is monodromic, therefore, it has a center at the origin and the sufficiency conditions follows.

We prove the necessity by reduction to absurdity. Assume that not all the coefficients $\alpha_{2 i}$ be null and take $N=\min \left\{i \in \mathbb{N}: \alpha_{2 i} \neq 0\right\}$, that is, $\alpha_{2 i}=0$ for all $i<N$ and $\alpha_{2 N} \neq 0$. Let $\mathbf{R}$ be the vector field associated with system (1.4) and $\mathbf{G}=\mathbf{F}_{n}+\left(\begin{array}{c}F\left(x^{2}, y\right) \\ x G\left(x^{2}, y\right)\end{array}\right):=\mathbf{F}_{n}+\sum_{j>n} \widetilde{\mathbf{G}}_{j}, \widetilde{\mathbf{G}}_{j} \in$ $\widetilde{\mathcal{Q}}_{j}^{\mathbf{t}}$ then $\mathbf{R}=\mathbf{G}+\nu \mathbf{D}_{0}$ with $\nu=\sum_{i \geq N} \alpha_{2 i} x^{2 i}$. Taking into account the origin of $\dot{\mathbf{x}}=\mathbf{G}(\mathbf{x})$ is a center, considering the decomposition (1.3) we have $\widetilde{\mathbf{G}}_{j}=\mathbf{X}_{\bar{h}_{j}}+\widetilde{\lambda}_{j} \mathbf{D}_{0}$, where $\bar{h}_{j} \in \overline{\mathcal{P}}_{j+2(n+1)}^{\mathbf{t}}$ and $\widetilde{\lambda}_{j} \in \widetilde{\mathcal{P}}_{j}^{\mathbf{t}}$ and considering that $\mathbf{F}_{n}=\mathbf{X}_{h}+\mu \mathbf{D}_{0}$ with $h=-\frac{1}{2}\left(y^{2}+(n+1) x^{2 n+2}\right)$ and $\mu=d x^{n}$, we get 


$$
\begin{aligned}
\mathbf{G} \cdot \mathbf{R}^{\perp} & =\mathbf{G} \wedge \nu \mathbf{D}_{0}=\nu\left(\mathbf{F}_{n}+\sum_{j>n} \tilde{\mathbf{G}}_{j}\right) \wedge \mathbf{D}_{0} \\
& =\nu\left(\mathbf{X}_{h}+\mu \mathbf{D}_{0}+\sum_{j>n}\left(\mathbf{X}_{\bar{h}_{j}}+\widetilde{\lambda}_{j} \mathbf{D}_{0}\right)\right) \wedge \mathbf{D}_{0} \\
& =x^{2 N}\left(\alpha_{2 N}+\sum_{l>0} \alpha_{2(l+N)} x^{2 l}\right)\left(2(n+1) h+\sum_{j>n}(2 n+2+j) \bar{h}_{j}\right) .
\end{aligned}
$$

In this way, for all $(x, y)$ in a neighborhood of the origin with $x \neq 0$, we have that $\operatorname{sig}\left(\mathbf{G} \cdot \mathbf{R}^{\perp}\right)=\operatorname{sig}\left(\alpha_{2(q+N+1)}\right)$ and for $x=0$ we get $\mathbf{G} \cdot \mathbf{R}^{\perp}=0$, therefore, all orbits of $\mathbf{R}$ leave from any closed orbits of the center $\mathbf{G}$ if $\alpha_{2 N}>0$ or all orbit of $\mathbf{R}$ enter from any closed orbit of the center $\mathbf{G}$ if $\alpha_{2 N}<0$. Therefore, the origin of system $\dot{\mathbf{x}}=\mathbf{F}(\mathbf{x})$ is not a center and this fact is a contradiction.

\section{Applications}

In this section, we solve the center problem using the method developed in previous sections.

First, we consider the real differential polynomial system

$$
\left(\begin{array}{c}
\dot{x} \\
\dot{y}
\end{array}\right)=\left(\begin{array}{c}
y+d x^{2} \\
-2 x^{3}+2 d x y
\end{array}\right)+\left(\begin{array}{c}
a_{30} x^{3}+a_{11} x y \\
b_{40} x^{4}+b_{21} x^{2} y+b_{02} y^{2}
\end{array}\right) .
$$

For such differential system, we can establish the next result.

Theorem 3.17. The origin of system (3.10) for $d \neq 0$ with $a_{11}+2 b_{02}=0$ is a center if and only if one of the following conditions hold:

(a) $b_{40}-5 b_{02}=a_{30}+2 b_{02} d=b_{21}+4 b_{02} d=0$;

(b) $b_{02}=b_{21}+3 a_{30}=a_{30} d+b_{40}=0$.

Moreover, all centers of system (3.10) with $d \neq 0$ are not analytically integrable.

Proof. First we study the necessity. If we apply the scaling of variables $x=\frac{u}{d}$ and $y=\frac{v}{d}$ system (3.10) is transformed to

$$
\left(\begin{array}{c}
\dot{u} \\
\dot{v}
\end{array}\right)=\left(\begin{array}{c}
v+u^{2} \\
-2 S u^{3}+2 u v
\end{array}\right)+\left(\begin{array}{c}
A_{30} u^{3}+A_{11} u v \\
B_{40} u^{4}+B_{21} u^{2} v+B_{02} v^{2}
\end{array}\right),
$$

where $S=1 / d^{2}, A_{30}=a_{30} / d^{2}, A_{11}=a_{11} / d, B_{40}=b_{40} / d^{3}, B_{21}=b_{21} / d^{2}$, and $B_{02}=b_{02} / d$. Next we reparameterize taking

$$
A_{30}=d_{0}-c_{1}, A_{11}=d_{1}-2 c_{2}, B_{40}=5 c_{0}, B_{21}=3 c_{1}+2 d_{0}, B_{02}=c_{2}+2 d_{1} \text {. }
$$

Now we compute the new normal form of system (3.10) transforming the system into the form (1.4) and the first necessary condition is given by

$$
\alpha_{2}=\frac{5\left(4 c_{2}+5 d_{0}-5 d_{1}\right) S+30 c_{0}-6 c_{1}+2 c_{2}+d_{0}-d_{1}}{25 S^{2}+1} .
$$


We have computed $\alpha_{2 i}$ for $i=1$ to 7 , but we do not write explicitly here the constants due their extension. To obtain the necessary condition for the existence of a center of system (3.10) we look for the irreducible decomposition of the variety $V(I)$ of the ideal $I=\left\langle\alpha_{2}, \ldots, \alpha_{14}\right\rangle$ over the field of rational numbers. One of the most efficient tools to find such irreducible decomposition is the routine minAssGTZ [41] of the computer algebra system SINGULAR [36]. The decomposition over the field of rational numbers consists of 13 components but as $S=1 / d^{2}>0$ the unique possible components are defined by the following cases:

(1) $c_{1}=d_{0}+2 c_{2}=2 c_{0}+d_{0} S=0$;

(2) $d_{0}=c_{2}=5 c_{0}-c_{1}=0$;

(3) $c_{2}=d_{0}-6 c_{1}+30 c_{0}+25 d_{0} S=6 c_{1}^{2}-5 c_{0} d_{0}-c_{1} d_{0}=0$.

Undoing the changes, cases (1) and (2) correspond to the cases (a) and (b) of Theorem 3.17, respectively. The last case (3) is not a true case because the conditions derive into complex roots.

Now we study the sufficiency. Under the assumptions of case (1) system ((3.11) has an inverse integrating factor of the form $V=\left(S u^{4}+v^{2}\right)\left(1-2 c_{2} v\right)$. Then by Theorem 1.3 of [9] system (3.11) has a center at the origin. Doing the change of variable $z=v+u^{2}-5 c_{0} u^{3}$ system (3.11) under the assumptions of case (2) takes the form

$$
\dot{z}=-u\left(2 u^{2}+2 S u^{2}-75 c_{0}^{2} u^{4}-4 z\right), \quad \dot{u}=z .
$$

System (3.12) is a time reversible system because it has the invariant symmetry $(z, u, t) \rightarrow(z,-u,-t)$; therefore, it has a center at the origin and consequently also system (3.11) and system (3.10).

Finally, all centers of the family (3.10) with $d \neq 0$ are not analytically integrable because the first quasi-homogeneous component $\mathbf{F}_{1}:=$ $\left(\begin{array}{c}y+\mathrm{d} x^{2} \\ -2 x^{3}+2 \mathrm{~d} x y\end{array}\right)$ with $d \neq 0$ is not polynomially integrable, see [13, Theorem 3.2$]$.

Finally, we study the center problem of the differential system

$$
\left(\begin{array}{c}
\dot{x} \\
\dot{y}
\end{array}\right)=\left(\begin{array}{c}
y \\
-x^{5}
\end{array}\right)+\left(\begin{array}{c}
a_{50} x^{5}+a_{21} x^{2} y \\
b_{70} x^{7}+b_{41} x^{4} y+b_{02} x y^{2}
\end{array}\right) .
$$

Theorem 3.18. The origin of system (3.13) is a center if and only if one of the following conditions holds:

(a) $a_{50}=b_{41}=0\left(R_{x}\right.$-reversible, $R_{y}$-reversible $)$

(b) $5 a_{50}+b_{14}=a_{21}+b_{12}=0$ (Hamiltonian)

Moreover, all centers of system (3.13) are analytically integrable.

Proof. First, we study the necessity. We compute the new normal form of system (3.13) and the first necessary condition is given by $\alpha_{4}=\frac{1}{8}\left(5 a_{50}+b_{41}\right)$. If we take condition $\alpha_{4}=0$, i.e. $b_{41}=-5 a_{50}$, then the second constant is $\alpha_{6}=\frac{3}{10} a_{50}\left(a_{21}+b_{12}\right)$. Now we consider the two possibilities for $\alpha_{6}=0$.

(i) If $a_{50}=0$ we obtain case (a). In this case, system (3.13) is $R_{x}$-reversible and $R_{y}$-reversible; therefore, it has a center at the origin. 
(ii) If $a_{21}+b_{12}=0$ we obtain case (b). In this case, system (3.13) is Hamiltonian; therefore, it has a center at the origin

In the case (a) system (3.13) is

$$
\left(\begin{array}{l}
\dot{x} \\
\dot{y}
\end{array}\right)=\left(\begin{array}{c}
y \\
-x^{5}
\end{array}\right)+\left(\begin{array}{c}
a_{21} x^{2} y \\
b_{70} x^{7}+b_{02} x y^{2}
\end{array}\right),
$$

and this system is $R_{x}$-reversible and $R_{y}$-reversible; therefore, if we apply the singular change of variable $X=x^{2}, Y=y^{2}$ and the rescaling in time $\mathrm{d} T=2 x y \mathrm{~d} t$, we obtain

$$
\left(\begin{array}{l}
X^{\prime} \\
Y^{\prime}
\end{array}\right)=\left(\begin{array}{c}
1+a_{21} X \\
-X^{2}+b_{70} X^{3}+b_{02} Y
\end{array}\right),
$$

where $/=\frac{\mathrm{d}}{\mathrm{d} T}$, and this system is analytically integrable applying the flow box theorem. Therefore, system (3.14) is analytically integrable.

In the case (b), system is analytically integrable since it is Hamiltonian. In consequence all centers of the family (3.13) are analytically integrable.

\section{Acknowledgements}

The first and second authors are partially supported by Ministerio de Ciencia, Innovación y Universidades/ FEDER grant number PGC2018-096265B-I00 and by the Consejería de Educación y Ciencia de la Junta de Andalucía (projects P12-FQM-1658, FQM-276). The third author is partially supported by a MINECO/ FEDER grant number PID2020-113758GB-I00 and an AGAUR (Generalitat de Catalunya) Grant number 2017SGR-1276.

Open Access. This article is licensed under a Creative Commons Attribution 4.0 International License, which permits use, sharing, adaptation, distribution and reproduction in any medium or format, as long as you give appropriate credit to the original author(s) and the source, provide a link to the Creative Commons licence, and indicate if changes were made. The images or other third party material in this article are included in the article's Creative Commons licence, unless indicated otherwise in a credit line to the material. If material is not included in the article's Creative Commons licence and your intended use is not permitted by statutory regulation or exceeds the permitted use, you will need to obtain permission directly from the copyright holder. To view a copy of this licence, visit http:// creativecommons.org/licenses/by/4.0/.

Publisher's Note Springer Nature remains neutral with regard to jurisdictional claims in published maps and institutional affiliations.

\section{References}

[1] Algaba, A., Checa, I., García, C., Gamero, E.: On orbital-reversibility for a class of planar dynamical systems. Commun. Nonlinear Sci. Numer. Simul. 20(1), 229-239 (2015)

[2] Algaba, A., Freire, E., Gamero, E., García, C.: Monodromy, center-focus and integrability problem for quasi-homogeneous polynomial systems. Nonlinear Anal. 72, 1726-1736 (2010) 
[3] Algaba, A., Fuentes, N., García, C.: Centers of quasi-homogeneous polynomial planar systems. Nonlinear Anal.-Real World Appl. 13, 419-431 (2012)

[4] Algaba, A., Gamero, E., García, C.: The integrability problem for a class of planar systems. Nonlinearity 22, 395-420 (2009)

[5] Algaba, A., Gamero, E., García, C.: The center problem. A view from the normal form theory. J. Math. Anal. Appl. 434, 680-697 (2016)

[6] Algaba, A., García, C., Giné, J.: Nilpotent centres via inverse integrating factors. Eur. J. Appl. Math. 27(5), 781-795 (2016)

[7] Algaba, A., García, C., Giné, J.: Nondegenerate and nilpotent centers for a cubic system of differential equations. Qual. Theory Dyn. Syst. 18(1), 333-345 (2019)

[8] Algaba, A., García, C., Giné, J.: Analytic integrability around a nilpotent singularity. J. Differ. Equ. 267, 443-467 (2019)

[9] Algaba, A., García, C., Giné, J.: Integrability of planar nilpotent differential systems through the existence of an inverse integrating factor. Commun. Nonlinear Sci. Numer. Simul. 71, 130-140 (2019)

[10] Algaba, A., García, C., Giné, J.: Center conditions of a particular polynomial differential system with a nilpotent singularity. J. Math. Anal. Appl. 483(2), 123639 (2020)

[11] Algaba, A., García, C., Giné, J., Llibre, J.: The center problem for $\mathbb{Z}_{2^{-}}$ symmetric nilpotent vector fields. J. Math. Anal. Appl. 466, 183-198 (2018)

[12] Algaba, A., García, C., Reyes, M.: The center problem for a family of systems of differential equations having a nilpotent singular point. J. Math. Anal. Appl. 340(1), 32-43 (2008)

[13] Algaba, A., García, C., Reyes, M.: Integrability of two dimensional quasihomogeneous polynomial differential system. Rocky Mt. J. Math. 41(1), 1-22 (2011)

[14] Algaba, A., García, C., Reyes, M.: Existence of an inverse integrating factor, center problem and integrability of a class of nilpotent systems. Chaos Solitons Fract. 45, 869-878 (2012)

[15] Algaba, A., García, C., Teixeira, M.A.: Reversibility and quasi-homogeneous normal forms of vector fields. Nonlinear Anal. 73, 510-525 (2010)

[16] Álvarez, M.J., Gasull, A.: Monodromy and stability for nilpotent critical points. Int. J. Bifur. Chaos Appl. Sci. Eng. 15(4), 1253-1265 (2005)

[17] Álvarez, M.J., Gasull, A.: Generating limit cycles from a nilpotent critical point via normal forms. J. Math. Anal. Appl. 318(1), 271-287 (2006)

[18] Andreev, A.F.: Solution to the problem of the center and the focus in one case. Akad. Nauk SSSR. Prikl. Mat. Meh. 17, 333-338 (1953)

[19] Andreev, A.F.: Investigation on the behaviour of the integral curves of a system of two differential equations in the neighborhood of a singular point. Trans. Am. Math. Soc. 8, 187-207 (1958)

[20] Andreev, A.F., Andreeva, I.A., Detchenya, L.V., Makovetskaya, T.V., Sadovskii, A.P.: Nilpotent centers of cubic systems. Differ. Equ. 53(8), 10031008 (2017)

[21] Baider, A., Sanders, J.: Further reduction of the Bogdanov-Takens normal form. J. Differ. Equ. 99, 205-244 (1992) 
[22] Berthier, M., Moussu, R.: Réversibilité et classification des centres nilpotents. Ann. Inst. Fourier (Grenoble) 44(2), 465-494 (1994)

[23] Bogdanov, R.I.: The versal deformation of a singular point of a vector field on the plane in the case of zero eigenvalues. Trans. Petrovski Sem. 2, 37-65 (1976). (in Russian)

[24] Bogdanov, R.I.: Local orbital normal form of vector fields on a plane. Trans. Petrovski Sem. 5, 50-85 (1979). (in Russian)

[25] Chavarriga, J., Giacomini, H., Giné, J., Llibre, J.: Local analytic integrability for nilpotent centers. Ergodic Theory Dyn. Syst. 23(2), 417-428 (2003)

[26] Chow, S.N., Hale, J.K.: Methods of Bifurcation Theory. Springer, New York (1982)

[27] Li, F., Liu, Y., Yuanyuan, Y., Yu, P.: Bi-center problem and bifurcation of limit cycles from nilpotent singular points in $\mathbb{Z}_{2}$-equivariant cubic vector fields. J. Differ. Equ. 265(10), 4965-4992 (2018)

[28] García, I.A.: Formal inverse integrating factor and the nilpotent center problem. Int. J. Bifur. Chaos Appl. Sci. Eng. 26(1), 1650015,13 (2016)

[29] García, I.A., Giacomini, H., Giné, J., Llibre, J.: Analytic nilpotent centers as limits of nondegenerate centers revisited. J. Math. Anal. Appl. 441(2), 893-899 (2016)

[30] Gasull, A., Torregrosa, J.: Center problem for several differential equations via Cherkas' method. J. Math. Anal. Appl. 228, 322-343 (1998)

[31] Giacomini, H., Giné, J., Llibre, J.: The problem of distinguishing between a center and a focus for nilpotent and degenerate analytic systems. J. Differ. Equ. 227, 406-426 (2006) (J. Differ. Equ. 232, 702, 2007, Corrigendum)

[32] Giné, J.: Sufficient conditions for a center at completely degenerate critical point. Int. J. Bifur. Chaos Appl. Sci. Eng. 12(7), 1659-1666 (2002)

[33] Giné, J.: The nondegenerate center problem and the inverse integrating factor. Bull. Sci. Math. 130(2), 152-161 (2006)

[34] Giné, J.: Center conditions for nilpotent cubic systems using the Cherkas method. Math. Comput. Simul. 129, 1-9 (2016)

[35] Giné, J., Llibre, J.: A method for characterizing nilpotent centers. J. Math. Anal. Appl. 413(1), 537-545 (2014)

[36] Greuel, G.M., Pfister, G., Schönemann, H.A.: Singular 3.0 A Computer Algebra System for Polynomial Computations. Centre for Computer Algebra, University of Kaiserlautern(2005). http://www.singular.uni-kl.de

[37] Lyapunov, A.M.: Stability of motion. In: Mathematics in Science and Engineering, vol. 30. Academic Press, New York (1966)

[38] Mañosa, V.: On the center problem for degenerate singular points of planar vector fields. Int. J. Bifurc. Chaos 12(4), 687-707 (2002)

[39] Mazzi, L., Sabatini, M.: A characterization of centres via first integrals. J. Differ. Equ. 76, 222-237 (1988)

[40] Moussu, R.: Symétrie et forme normale des centres et foyers dégénérés.(French) [Symmetry and normal form in degenerate centers and foci]. Ergod. Theory Dyn. Syst. 2(2), 241-251 (1983)

[41] Pfister, G., Decker, W., Schönemann, H., Laplagne, S.: primdec.lib. A singular 3.0 library for computing primary decomposition and radical of ideals (2005) 
[42] Poincaré, H.: Mémoire sur les courbes définies par les équations différentielles. J. Math. 7, 375-422 (1881) (8 (1882), 251-296; Oeuvres de Henri Poincaré, vol. I, Gauthier-Villars, Paris, 1951, pp. 3-84)

[43] Poincaré, H.: Mémoire sur les courbes définies par les équations différentielles. J. Math. 3, 375-422 (1881) (8 (1882), 251-296; Oeuvres de Henri Poincaré, vol. I, Gauthier-Villars, Paris, 1951, pp. 3-84)

[44] Poincaré, H.: Oeuvres de Henri Poincaré, vol. I, pp. 95-114. Gauthier-Villars, Paris (1951)

[45] Sadovski, A.P.: Formal orbital normal forms of two-dimensional systems with non-zero linear part. Differ. Equ. 21(9), 1509-1516 (1965)

[46] Takens, F.: Singularities of vector fields. Inst. Hautes Études Sci. Publ. Math. 43, 47-100 (1974)

[47] Tang, Y., Li, W., Zhang, Z.: Focus-center problem of planar degenerate system. J. Math. Anal. Appl. 345(2), 934-940 (2008)

Antonio Algaba and Cristóbal García

Department Ciencias Integradas, Centro de Estudios Avanzados en Física Matemáticas y Computación, Facultad de Ciencias

University of Huelva

Huelva

Spain

e-mail: algaba@uhu.es

Cristóbal García

e-mail: cristoba@uhu.es

Jaume Giné

Departament de Matemàtica

Universitat de Lleida

Av. Jaume II, 69

25001 Lleida Catalonia

Spain

e-mail: jaume.gine@udl.cat

Received: March 12, 2020.

Revised: November 16, 2020.

Accepted: June 3, 2021. 